\title{
Universal Evolutionary Model for Periodical Species
}

\author{
Eric Goles $\mathbb{D},{ }^{1}$ Ivan Slapnicar $\mathbb{D}^{2},{ }^{2}$ and Marco A. Lardies $\mathbb{D}^{3}$ \\ ${ }^{1}$ Facultad de Ingenieria y Ciencias, Universidad Adolfo Ibañez, Diagonal Las Torres 2700, Peñalolen, Santiago, Chile \\ ${ }^{2}$ University of Split, Faculty of Electrical Engineering, Mechanical Engineering and Naval Architecture, R. Boškovića 32, \\ 21000 Split, Croatia \\ ${ }^{3}$ Facultad de Artes Liberales, Universidad Adolfo Ibañez, Santiago, Chile
}

Correspondence should be addressed to Eric Goles; antonio.chacc@gmail.com

Received 22 June 2021; Accepted 22 September 2021; Published 11 October 2021

Academic Editor: Dan Selişteanu

Copyright (c) 2021 Eric Goles et al. This is an open access article distributed under the Creative Commons Attribution License, which permits unrestricted use, distribution, and reproduction in any medium, provided the original work is properly cited.

Real-world examples of periodical species range from cicadas, whose life cycles are large prime numbers, like 13 or 17 , to bamboos, whose periods are large multiples of small primes, like 40 or even 120. The periodicity is caused by interaction of species, be it a predator-prey relationship, symbiosis, commensalism, or competition exclusion principle. We propose a simple mathematical model, which explains and models all those principles, including listed extremal cases. This rather universal, qualitative model is based on the concept of a local fitness function, where a randomly chosen new period is selected if the value of the global fitness function of the species increases. Arithmetically speaking, the different interactions are related to only four principles: given a couple of integer periods either (1) their greatest common divisor is one, (2) one of the periods is prime, (3) both periods are equal, or (4) one period is an integer multiple of the other.

\section{Introduction}

Life-history diversity is a remarkable feature of living species and underlies fundamental evolutionary questions [1]. Periodical species are well known for their mass and synchronous reproduction [2]. The use of an appropriate model to evaluate synchronicity in periodical species depends first on the life-history strategies of the species. For example, some species have a life-history with discrete nonoverlapping generations; that is, there are no adult survivors from one generation to the next. Such species includes annual plants, annual insects, salmon, periodical cicadas, and bamboos species [3]. Once the adults reproduce, they perish, and the future of the population is based on the dormant or juvenile stage of the organism. In periodical species, the population emerges synchronously from the ground or benthos (cicadas, bamboos, red or brown tides of phytoplankton species) as juveniles or adults. The growth rates before emerging in periodical cycle species are obviously affected by the environment being the main driver for maintaining a minimum viable population (for example, extinction probability), the interspecific interactions with predators (including parasitoids), symbiosis, interspecific competition, and/or disturbances in the habitat [4-7]. Furthermore, the nature of these interactions can vary depending on the evolutionary context and environmental conditions in which they occur [8]; nevertheless, the synchrony is maintained despite abrupt changes in the environment $[9,10]$.

Dilucidating, in which natural forces drive these extraordinary periodical cycles, is a central challenge in ecology. To understand these evolutionary processes, a broader view is needed of the properties of multiscale spatiotemporal patterns in species-environment interactions [11]. Periodical species are plants or animals that emerge in nature every $T>1$ years, like some few species of cicadas (every 3,13 , and 17 years) or bamboos $(2,3,5,15,32$, 60 , and 120 years), and other flowering plants [12]. Densitydependent selection is the simplest form of feedback between an ecological effect of an organism's own making (crowding due to sustained population growth) and the selective response to the resulting conditions [13]. Densitydependent fitness and density-dependent selection are critical concepts underlying ideas about adaptation to biotic 
selection pressures and the coadaptation of interacting species. To understand those periods (and other related processes), several models have been proposed [14]. In most of them, one of the relevant assumptions is the "satiation hypothesis"; that is, organisms have to emerge in very large numbers in order to satiate predators (birds, rats, etc.), which is consequently linked to climate conditions $[12,15]$ but also to reduction in ecosystem productivity [10]. Those assumptions explain synchronicity but not necessarily $T>1$ life cycles. Since the usual life cycle of those organisms is annual, the question is how it may evolve to a $T>1$ period? Several evolutionary pressures have been proposed to explain periodical life cycles. Some years ago, a deterministic discrete populational model was presented as a mechanism that is related with systematic low temperatures in the ice ages in Pleistocene $[16,17]$. In this case, under the cold temperature pressure, selected cicadas are those with large periods underground during the metamorphosis stage in order to avoid low temperatures (but see also [18]). Other theories regarding stabilizing selection provide explanations for the existence of synchronous seeding in bamboos [19]. From this latter assumption, they proposed a model such that, from the usual synchronously annual emergence of seeds, a mutation with period $T>1$ may appear. However, some problems remained: Why periodical cicadas have prime life cycles? Is discrete multiplication the explanation that some flowering intervals evolved in bamboos have small prime cycles while some have very large nonprimes life cycles? Some authors have proposed a very simple model for cicadas prime life cycles by assuming the existence of a periodical predator [20]. In this context, it was proved that the fixed points for the nonextinct periodical cicadas are necessarily prime numbers. Nevertheless, simulations were done, which exhibit convergence to prime numbers only by accepting mutations in a narrow temporal scale (for instance \pm one year concerning the current life cycle). Other models take into account an hybridization hypothesis, which entails that life cycle evolution has a phylogenetic component, where evolution of periodicity in species was due to glacial cooling during the Pleistocene and the selection for primenumbered cycles via hybrid breakdown of nonprimenumbered cycles [17]. That is, if the life cycle of two species of prey is prime numbers, $T$ and $T^{\prime}$, so that they encounter only every multiple of $T \times T^{\prime}$ years, this diminishes drastically the probability of hybridization, which will produce other life cycles with less offspring [16]. However, it is important to remark that, in order to satisfy the hybridization hypothesis, it is enough to consider only relative primes life cycles [14]. Based on the several gaps of the models presented previously, it is required to develop a mathematical theory that includes the possible mechanism of how periodical life cycles are shaped by diverse kinds of interspecific interactions in an evolutionary context in nature.

In this work, we propose a universal qualitative framework for species with synchronic periodical cycles, which is based on local fitness functions and the evolution of species interaction evaluating the extinction probability of species. Additionally, we describe the convergence of the proposed model and completely characterize its fixed points, leading to the conclusion that different interactions are related to only four principles. The paper is organized as follows: in section General Model, we define general model based on the notion of a fitness function. In section Classes of Fitness Functions, we classify all fitness functions that appear in our model and reduce them to 18 cases (fitness tuples), which need to be studied. In section Dynamics of the Model, we describe the dynamics of the model and state the evolution algorithm. In section Characterization of Fixed Points, we characterize all fixed points of the studied fitness functions in the case of bounded mutations. In section Numerical Simulations, we present numerical simulations for two cases of bounded mutations as well as more general "quantitative" fitness functions. In section Discussion, we discuss our results and some open questions, including small modifications with fixed points, which are very large periods. In section Conclusion, we draw our conclusions.

\section{Model}

2.1. General Model. Consider two species, $\mathscr{C}_{1}$ and $\mathscr{C}_{2}$, with an emergence period $c_{1}, c_{2} \in \mathbb{N}, c_{1}, c_{2} \geq 2$ (in years). Consider the interval $T=c_{1} \cdot c_{2}$, and the emergence functions

$$
\chi_{i}:[1, T] \longrightarrow\{0,1\}
$$

such that $\chi_{i}(t)=1$ if and only if the species $\mathscr{C}_{i}$ emerges in year $t$. Clearly, species $\mathscr{C}_{1}$ emerges exactly $c_{2}$ times in the interval $[1, T]$ and, respectively, $\mathscr{C}_{2}$ emerges exactly $c_{1}$ times.

Let us now define the (local) fitness functions associated to $\mathscr{C}_{1}$ and $\mathscr{C}_{2}$ as

$$
f_{i}:\{0,1\} \times\{0,1\} \longrightarrow\{-1,0,1\}, \quad i=1,2,
$$

such that $f_{1}(0, *)=f_{2}(*, 0)=0$; that is, if species is dormant, the value of its fitness function is zero. If the species is emergent, the value of its fitness function with respect to the other species may be 1,0 or -1 (good, neutral or bad). In biological terms, this fitness function is an expected fitness, the probability of success given each periodical species' emergence. Furthermore, expected fitness is directly related to per capita population growth rate; that is, if fitness function is 1 , population increases in size; if function is 0 , population maintains its size; and if the fitness function is -1 , population decreases in size to extinction. This simple model is qualitative, but it captures all interesting types of behavior.

For given periods $c_{1}$ and $c_{2}$, the global (cumulative) fitness function over the interval $[1, T]$ is obtained by simply adding yearly values of local fitness function and dividing them by the number of years, in which the species appears in this interval:

$$
\begin{aligned}
& F_{1}\left(c_{1}, c_{2}\right)=\frac{1}{c_{2}} \sum_{t=1}^{T} f_{1}\left(\chi_{1}(t), \chi_{2}(t)\right), \\
& F_{2}\left(c_{1}, c_{2}\right)=\frac{1}{c_{1}} \sum_{t=1}^{T} f_{2}\left(\chi_{1}(t), \chi_{2}(t)\right) .
\end{aligned}
$$


2.2. Classes of Fitness Functions. Given two species, $\mathscr{C}_{1}$ and $\mathscr{C}_{2}$, and their local fitness functions, $f_{1}$ and $f_{2}$, respectively, the only interesting situations are when the species are emergent. Therefore, the only cases, which need to be considered, are given by the 4 -tuple

$$
v \equiv\left(v_{1}, v_{2}, v_{3}, v_{4}\right)=\left(f_{1}(1,0), f_{2}(0,1), f_{1}(1,1), f_{2}(1,1)\right) \in\{-1,0,1\}^{4}
$$

We shall call such 4-tuple fitness tuple or just tuple. Consequently, we have a total of $3^{4}=81$ possibly different tuples $v$. We reduce this number in three steps to only 18 tuples that need to be studied as follows. First, we eliminate equivalent tuples (two tuples are equivalent when they are obtained by simply exchanging species $\mathscr{C}_{1}$ and $\mathscr{C}_{2}$, that is $\left.\left(v_{1}, v_{2}, v_{3}, v_{4}\right) \equiv\left(v_{2}, v_{1}, v_{4}, v_{3}\right)\right)$. Then, we are left with $v$ to 45 tuples, which we divide into four classes (for the class $D$ we also take into account the symmetry when one exchanges species, that is, $(a, b, c, d)=(b, a, d, c))$ :

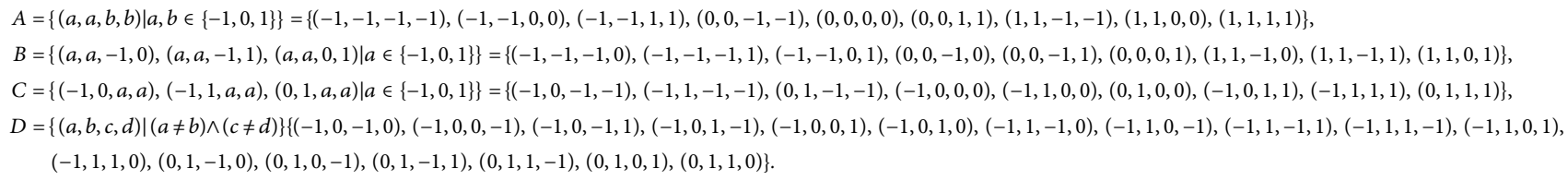

In the second step, we eliminate tuples without biological interest or meaning:

$$
\begin{aligned}
& (-1,-1,-1,-1), \\
& (-1,-1,0,0) \\
& (0,0,-1,-1) \\
& (0,0,0,0) \\
& (1,1,0,0) \\
& (1,1,1,1) \\
& (-1,-1,-1,0) \\
& (-1,-1,-1,1) \\
& (-1,-1,0,1) \\
& (0,0,-1,0) \\
& (0,0,0,1) \\
& (-1,0,-1,-1) \\
& (-1,1,-1,-1) \\
& (-1,0,0,0) \\
& (-1,1,0,0) \\
& (0,1,0,0) \\
& (0,1,1,1) \\
& (-1,0,-1,0) \\
& (-1,0,-1,1) \\
& (-1,1,-1,0) \\
& (-1,1,-1,1) \\
& (0,1,0,1)
\end{aligned}
$$

For example, for the tuple $(-1,0,-1,0)$, the species does not have any positive evolution pressure, and the other species is completely indifferent.

In the third step, we eliminate tuples, for which one of the fitness functions is always negative according to the following lemma:

Lemma 1. For the tuples

$$
\begin{aligned}
& (0,1,-1,-1), \\
& (0,0,-1,1), \\
& (0,1,-1,1), \\
& (0,1,-1,0), \\
& (0,-1,-1,0), \\
& (0,1,-1,0), \\
& (-1,0,1,-1),
\end{aligned}
$$

one of the global fitness functions is always negative.

See Appendix.

Therefore, the final set of tuples to study, $\mathscr{V}$, consists of the 18 tuples listed in Table 1. We call such tuples relevant fitness tuples and we number them for further reference.

Each tuple may be represented by its respective ecological graph as in Figure 1. By inspecting corresponding ecological graphs, we classify tuples from Table 1 in four classes. The first class, $\mathscr{V}_{1}$, is the set of all tuples, for which the corresponding graph is not strongly connected:

$$
\begin{aligned}
\mathscr{V}_{1} & =\left\{v \in \mathscr{V} \mid\left(f_{1}(1,1)=0\right) \vee\left(f_{2}(1,1)=0\right)\right\} \\
& =\left\{v_{7}, v_{10}, v_{11}, v_{13}, v_{14}, v_{15}, v_{16}, v_{17}, v_{18}\right\} .
\end{aligned}
$$

The other three classes are 


$$
\begin{aligned}
& \mathscr{V}_{2}=\left\{v \in \mathscr{V} \mid f_{1}(1,1)=f_{2}(1,1)=1\right\}=\left\{v_{1}, v_{2}, v_{3}, v_{4}, v_{5}\right\} \\
& \mathscr{V}_{3}=\left\{v \in \mathscr{V} \mid f_{1}(1,1)=f_{2}(1,1)=-1\right\}=\left\{v_{6}\right\} \\
& \mathscr{V}_{4}=\left\{v \in \mathscr{V} \mid f_{1}(1,1) \cdot f_{2}(1,1)=-1\right\}=\left\{v_{8}, v_{9}, v_{12}\right\} .
\end{aligned}
$$

2.3. Dynamics of the Model. We describe the evolution of species $\mathscr{C}_{1}$ and $\mathscr{C}_{2}$. Given initial periods $c_{1}, c_{2} \in \mathbb{N}, c_{1}, c_{2} \geq 2$, the first iteration of the evolution game is as follows: for the species $\mathscr{C}_{1}$ we propose random local change of its period $c_{1}$ to new period $c_{1}^{\prime} \in \mathbb{N}, c_{1}^{\prime} \geq 2$. If the value of the global fitness function of the species $\mathscr{C}_{1}$ has improved, that is, if $F_{1}\left(c_{1}^{\prime}, c_{2}\right)>F_{1}\left(c_{1}, c_{2}\right)$, the period $c_{1}^{\prime}$ is accepted as the new period, $c_{1} \leftarrow c_{1}^{\prime}$, and the value of the global fitness function of the other species, $\mathscr{C}_{2}$, is recomputed. The analogous procedure is now applied to the species $\mathscr{C}_{2}$. Such iterations are repeated until there is no change. The pseudocode of the algorithm is given in Algorithm 1.

We will also assume that the evolutionary game changes each species in a mutation interval $[-p, p]$ $=[-p,-p+1, \ldots,-1,0,1, \ldots, p]$; that is, the arbitrarily far moves do not exist in the algorithm. More precisely, the new periods $c_{1}^{\prime}$ and $c_{2}^{\prime}$ are chosen as follows:

$$
\begin{aligned}
c_{1}^{\prime} & =c_{1}+k, \\
c_{2}^{\prime} & =c_{2}+l, \\
k, l & \in[-p, p] .
\end{aligned}
$$

We say that a couple of periods $\left(c_{1}, c_{2}\right)$ for the given tuple $v$ are a $p$-fixed point if and only if $\forall k, l \in[-p, p]$

$$
\begin{gathered}
F_{1}\left(c_{1}+k, c_{2}\right) \leq F_{1}\left(c_{1}, c_{2}\right), \\
F_{2}\left(c_{1}, c_{2}+l\right) \leq F_{2}\left(c_{1}, c_{2}\right) .
\end{gathered}
$$

In this context, fixed points are related to the interval of possible time jumps. We also speak of global fixed point when the above is true for any $p \in \mathbb{N}$.

2.4. Characterization of Fixed Points. For the next results, we will consider the evolution algorithm for $p=1$, that is, in $[-1,1]$ mutation space. In particular, by "local fixed point" we mean 1-fixed point. The results for $p$-fixed points are similar (see Numerical Simulations and Discussion).

The first results are related with the relations between the sets of fixed points in any of the classes $\mathscr{V}_{i}, i=1,2,3,4$. For that, let us define the set of fixed points as follows:

$$
\mathscr{A}_{k}=\left\{\left(c_{1}, c_{2}\right) \mid c_{1}, c_{2} \in \mathbb{N} \text {, and it is an fixed point for the tuple } v_{k}\right\} \text {. }
$$

The relations between the sets are as follows.

Lemma 2. For the tuples in the class $\mathscr{V}_{1}$ we have
TABLE 1: Relevant fitness tuples.

\begin{tabular}{lcc}
\hline$v_{1}=(-1,0,1,1)$ & $v_{2}=(-1,1,1,1)$ & $v_{3}=(0,1,1,1)$ \\
$v_{4}=(0,0,1,1)$ & $v_{5}=(-1,-1,1,1)$ & $v_{6}=(1,1,-1,-1)$ \\
$v_{7}=(1,1,-1,0)$ & $v_{8}=(1,1,1,-1)$ & $v_{9}=(-1,1,1,-1)$ \\
$v_{10}=(-1,1,1,0)$ & $v_{11}=(1,0,-1,0)$ & $v_{12}=(0,1,1,-1)$ \\
$v_{13}=(0,1,1,0)$ & $v_{14}=(1,1,1,0)$ & $v_{15}=(0,-1,1,0)$ \\
$v_{16}=(-1,0,1,0)$ & $v_{17}=(1,-1,-1,0)$ & $v_{18}=(1,-1,1,0)$ \\
\hline
\end{tabular}

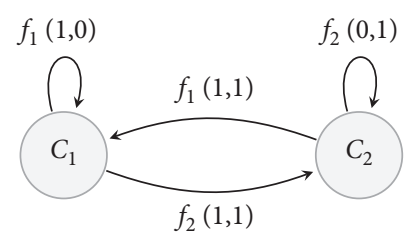

Figure 1: General ecological graph, where two emergent species, $\mathscr{C}_{1}$ and $\mathscr{C}_{2}$, interact with local fitness functions and both species coexist.

$$
\begin{aligned}
& \mathscr{A}_{7} \subsetneq \mathscr{A}_{11}, \\
\mathscr{A}_{10}= & \mathscr{A}_{13} \subsetneq \mathscr{A}_{14}, \\
& \mathscr{A}_{15} \subsetneq \mathscr{A}_{16}, \\
& \mathscr{A}_{10} \subsetneq \mathscr{A}_{16}, \\
& \mathscr{A}_{17} \subsetneq \mathscr{A}_{11}, \\
& \mathscr{A}_{17} \subsetneq \mathscr{A}_{18} .
\end{aligned}
$$

For the tuples in the class $\mathscr{V}_{2}$ we have

$$
\mathscr{A}_{1}=\mathscr{A}_{4}=\mathscr{A}_{5} \subsetneq \mathscr{A}_{2}=\mathscr{A}_{3} \text {. }
$$

For the tuples in the class $\mathscr{V}_{4}$ we have

$$
\mathscr{A}_{9}=\mathscr{A}_{12} \subsetneq \mathscr{A}_{8} \text {. }
$$

See Appendix.

Using Lemma 2, we eliminate tuples $v_{3}, v_{4}, v_{5}, v_{12}$ and $v_{13}$ from Table 1. We shall call the remaining tuples inside each class representative tuples. Now, we characterize the fixed points inside each class.

Proposition 1. For the representative tuples in the class $\mathscr{V}_{1}$ the structure of fixed points is as follows:

(i) For $v_{7}$ there exist global fixed points such that $\operatorname{gcd}\left(c_{1}, c_{2}\right)=1$, and there exist infinitely many local ones.

(ii) For $v_{10}$ there exist infinitely many local fixed points, but no global ones.

(iii) For $v_{11}$ there exist global fixed points such that $\operatorname{gcd}\left(c_{1}, c_{2}\right)=1$, as well as infinitely many local ones.

(iv) For $v_{14}$ there exist global fixed points such that $\operatorname{gcd}\left(c_{1}, c_{2}\right)=1$, as well as infinitely many local ones.

(v) For $v_{15}$, the couples $\left(c_{1}, c_{2}\right)$ such that $c_{1}=c_{2}$ are global fixed points. Further, there exist infinitely many local ones. 


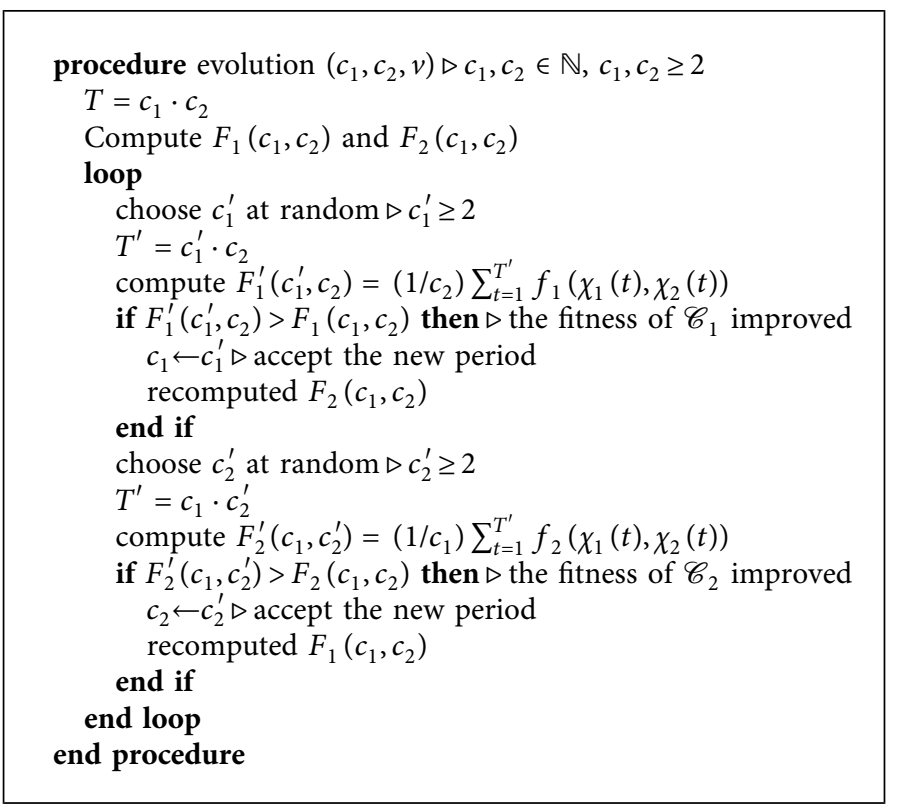

Algorithm 1: Evolution dynamics.

(vi) For $v_{16}$, the couples $\left(c_{1}, c_{2}\right)$ such that $c_{2} \mid c_{1}$ are global fixed points, and there exist infinitely many local ones.

(vii) For $v_{17}$ there are no global fixed points, but there are infinitely many local ones. For instance, couples $\left(c_{1}, c_{2}\right)$ such that $c_{1}$ is a prime number and $c_{2}<c_{1}-$ 1 are local fixed points.

(viii) For $v_{18}$, the couples $\left(c_{1}, c_{2}\right)$ such that $c_{1} \mid c_{2}$ are global fixed points, and there exist infinitely many local ones.

See Appendix.

Proposition 2. For the representative tuples in the class $\mathscr{V}_{2}$ we have

(i) $v_{1}$ admits the global fixed points $\left(c_{1}, c_{2}\right)$ such that $c_{1}=c_{2}$. Further, there exist also infinitely many local fixed points.

(ii) For $v_{2}$, any couple $\left(c_{1}, c_{2}\right)$ such that $c_{2} \mid c_{1}$ is a global fixed point. Further, there exist infinitely many local fixed points.

See Appendix.

Proposition 3. For the class $\mathscr{V}_{3}=\left\{v_{6}\right\}$, every $\left(c_{1}, c_{2}\right)$ such that $\operatorname{gcd}\left(c_{1}, c_{2}\right)=1$, is a global fixed point. There exist infinitely many local fixed points.

See Appendix.

Proposition 4. For the representative tuples in the class $\mathscr{V}_{4}$ we have

(i) $v_{8}$ admits global fixed points $\left(c_{1}, c_{2}\right)$ such that $\operatorname{gcd}\left(c_{1}, c_{2}\right)=1$. Further, it admits infinitely many local fixed points. (ii) $v_{9}$ only admits local fixed points: if $\left(c_{1}, c_{2}\right)$ is such that $c_{2}$ is prime and $c_{2} \notin\left\{c_{1}-1, c_{1}+1\right\}$, then it is a local fixed point. Further, the species $\mathscr{C}_{1}$ disappears. Also, there exist other infinite families of local fixed points.

See Appendix.

\section{Numerical Simulations}

We ran simulations to illustrate the behavior of the representative 4 -tuples. A couple of periods $\left(c_{1}, c_{2}\right)$ are a $p$-fixed point under simulation if, after thirty steps, neither of the species changes its period. We plot fixed points using the following convention: if $\left(c_{1}, c_{2}\right)$ is not a fixed point, the corresponding square on the plot is white; otherwise, it is red if both species survive, or gray if the species $\mathscr{C}_{1}$ disappears. The link to the complete code used to run simulations is given in Declarations.

The plots in Figures 2-7 depict the characteristic behavior of the qualitative model described in Propositions 1-4 (see also Discussion). For easier visual reference when interpreting the figures, Figure 8 shows canonical plots of three typical situations.

The first plot in each row shows the results of the simulation for the corresponding fitness tuple for the mutation interval $[-p, p]=[-1,1]$. The second plot in each row shows the results for the mutation interval $[-p, p]=[-4,4]-$ as expected, by increasing mutation interval, some local fixed points cease to be fixed points, but global fitness points remain.

We also simulated what happens in more "qualitative" setting, that is, when local fitness functions are generalized such that

$$
f_{i}:\{0,1\} \times\{0,1\} \longrightarrow \mathbb{R}, \quad i=1,2 .
$$



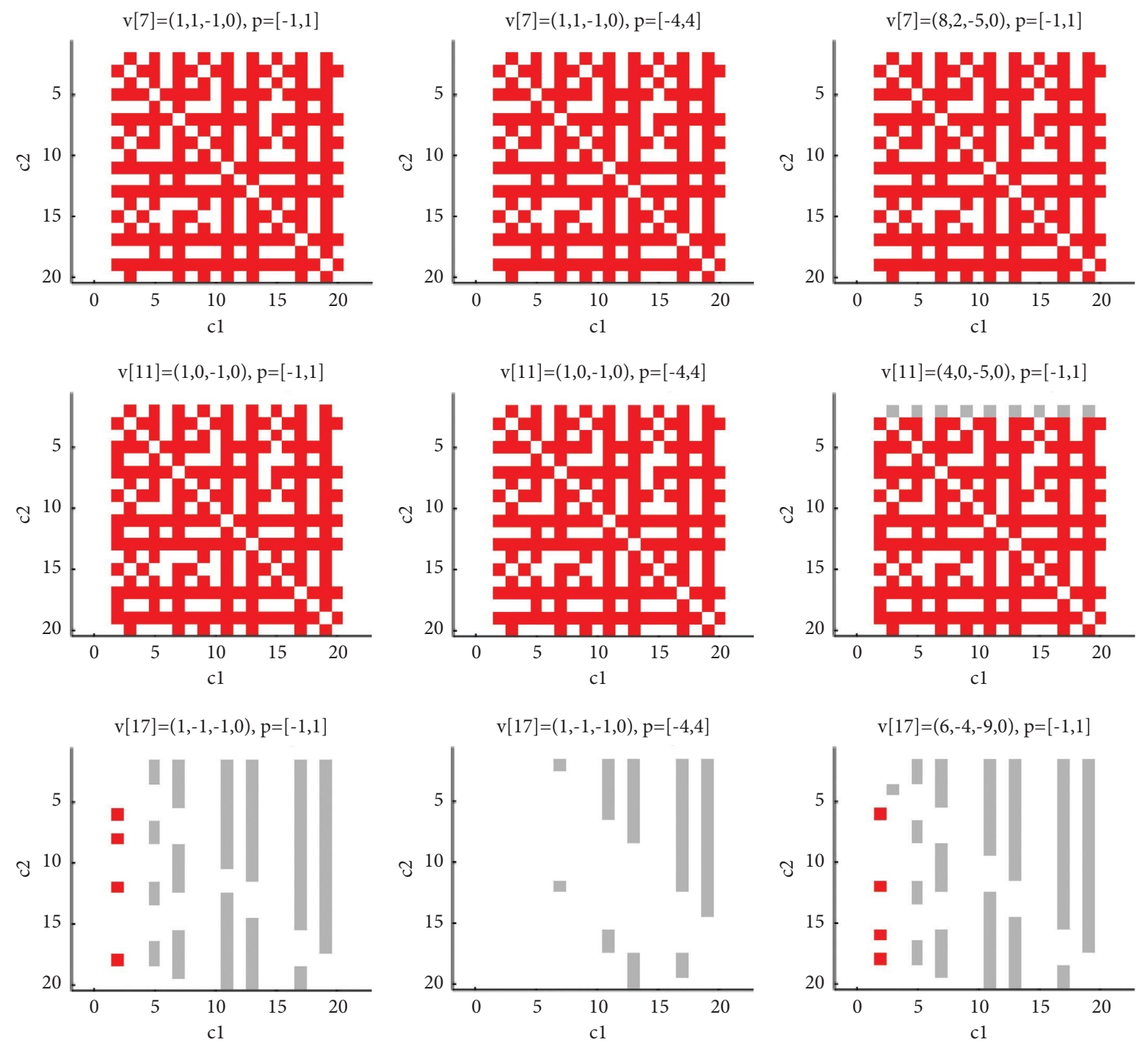

FIgURE 2: Attractors for tuples $v_{7}, v_{11}$ and $v_{17}$ from Proposition 1. The tuples are of a "competition" type. Global fixed points for $v_{7}$ and $v_{11}$ are those with $\operatorname{gcd}\left(c_{1}, c_{2}\right)=1$. In $v_{17}$, the species $\mathscr{C}_{1}$ has a behavior like periodic cicadas with prime number fixed points. In this case, the species $\mathscr{C}_{2}$ disappears, since the fitness $F_{2}\left(c_{1}, c_{2}\right)$ is always negative. Attractors are marked with red squares if both species survive and with gray squares if one of the species disappears.

In order to run these tests, each entry of fitness tuple was multiplied by a random integer from the interval $[1,10]$ and the mutation interval was $[-p, p]=[-1,1]$. The results are displayed in the third plot in each row. It is worth noting that the plots are indeed similar to the plots in the first two columns. These simulations indicate that our qualitative model covers well different qualitative cases, but this should by no means be considered a proof of such claim, without further analysis. Clearly, the analysis for any particular fitness tuple could be done if the specific case of interest appears.

\section{Discussion}

By analyzing the ecological factors that shape development, reproduction, and survival, the life-history theory seeks to explain the evolution of the major features of life cycles [21].
The complex spatial and temporal populational dynamics of periodical species are largely consequences of evolution of species interactions $[22,23]$. Our models are relatively simple and describe accurately the evolution of several types of periodical species with synchronized emergence including species associated with prime-numbered (i.e., insects) and nonprime-numbered life cycles (i.e., plants) [24-26]. Several fitness tuples in our model show that various life cycles and their respective periodical emergences extinguish each other. On the other hand, our computer simulations demonstrate that the evolution of the periodicity is possible under restricted conditions. Here, we first comment four propositions and their respective classes of fitness tuples.

In the class $\mathscr{V}_{1}$ (Proposition 1), there are two types of behavior. The tuples $v_{7}, v_{11}$ and $v_{17}$ are of a "competition" type. In $v_{17}$, the species $\mathscr{C}_{1}$ has a behavior like periodic cicadas with prime number fixed points (and in this case, the 

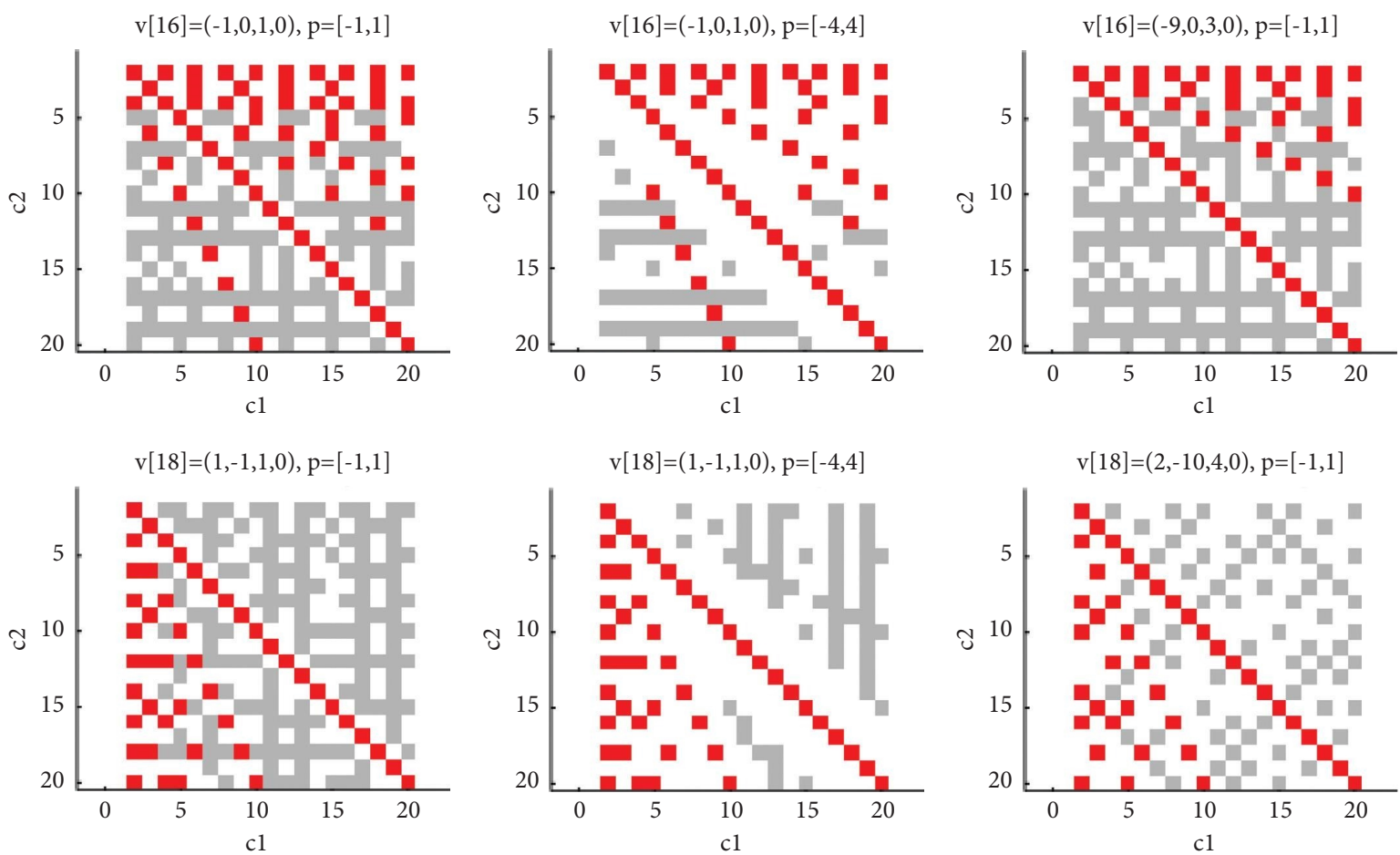

Figure 3: Attractors for tuples $v_{16}$ and $v_{18}$ from Proposition 1 . Here, one of the global fitness functions is constant $\left(F_{2}\left(c_{1}, c_{2}\right)=0\right.$ for $v_{16}$ and $F_{1}\left(c_{1}, c_{2}\right)=1$ for $\left.v_{18}\right)$, while the other species has a bamboo-like behavior-it emerges as an integer multiple of the constant one. In both cases, there is a "commensalism" interaction.

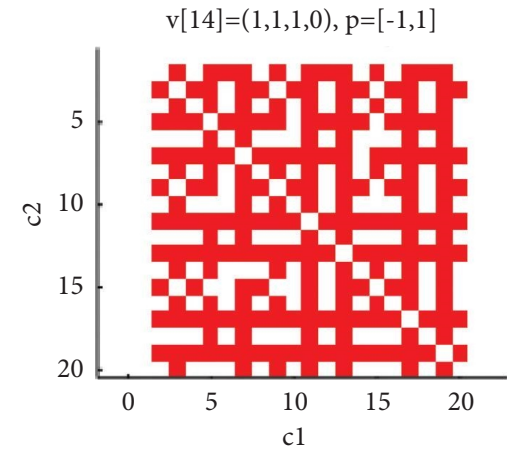

$\mathrm{v}[15]=(0,-1,1,0), \mathrm{p}=[-1,1]$

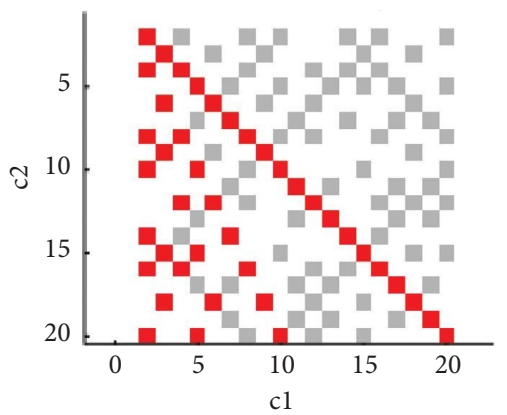

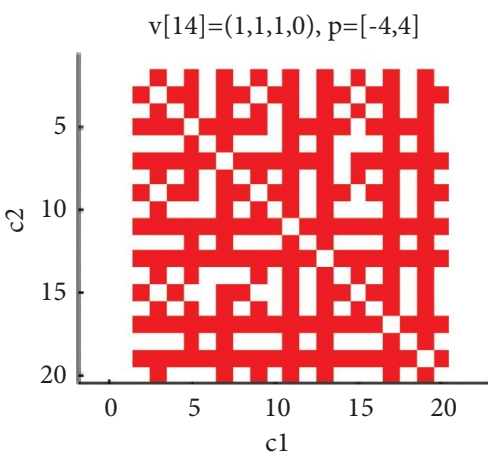

$\mathrm{v}[15]=(0,-1,1,0), \mathrm{p}=[-4,4]$

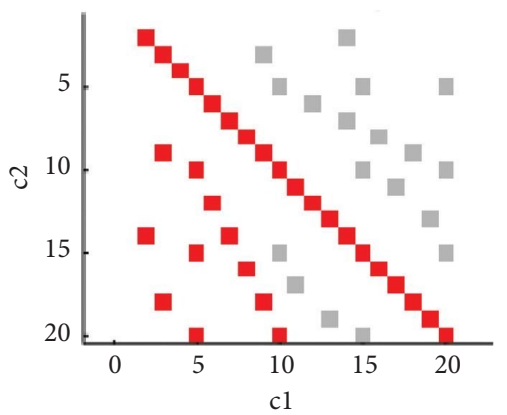

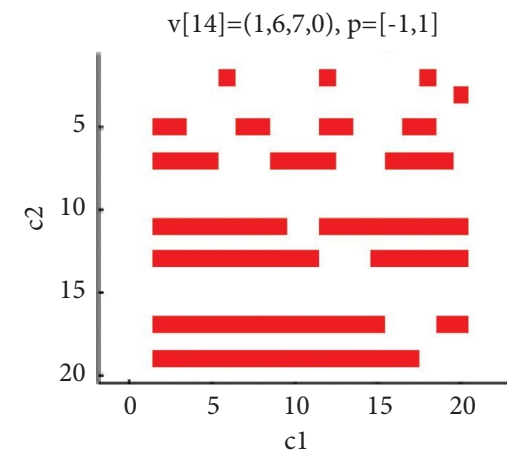

$\mathrm{v}[15]=(0,-2,10,0), \mathrm{p}=[-1,1]$

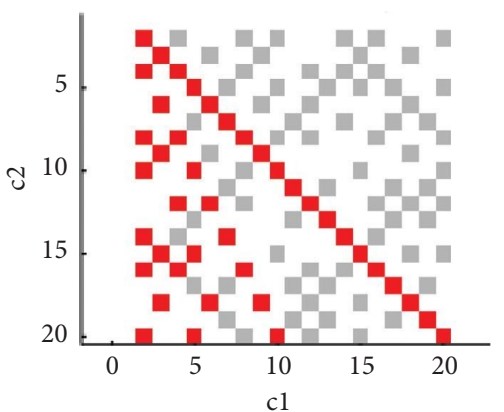

Figure 4: Attractors for tuples $v_{14}$ and $v_{15}$ from Proposition 1. For $v_{1}$, global fixed points are again those with $\operatorname{gcd}\left(c_{1}, c_{2}\right)=1$, and for $v_{15}$, global fixed points are those with $c_{1}=c_{2}$.

species $\mathscr{C}_{2}$ disappears, since the fitness $F_{2}\left(c_{1}, c_{2}\right)$ is always negative). The other type of behavior is the one generated by the tuples $v_{16}$ and $v_{18}$. Here, one of the global fitness functions is constant $\left(F_{2}\left(c_{1}, c_{2}\right)=0\right.$ for $v_{16}$ and $F_{1}\left(c_{1}, c_{2}\right)=$ 1 for $v_{18}$ ), while the other species has a bamboo-like behavior-it emerges as an integer multiple of the constant 

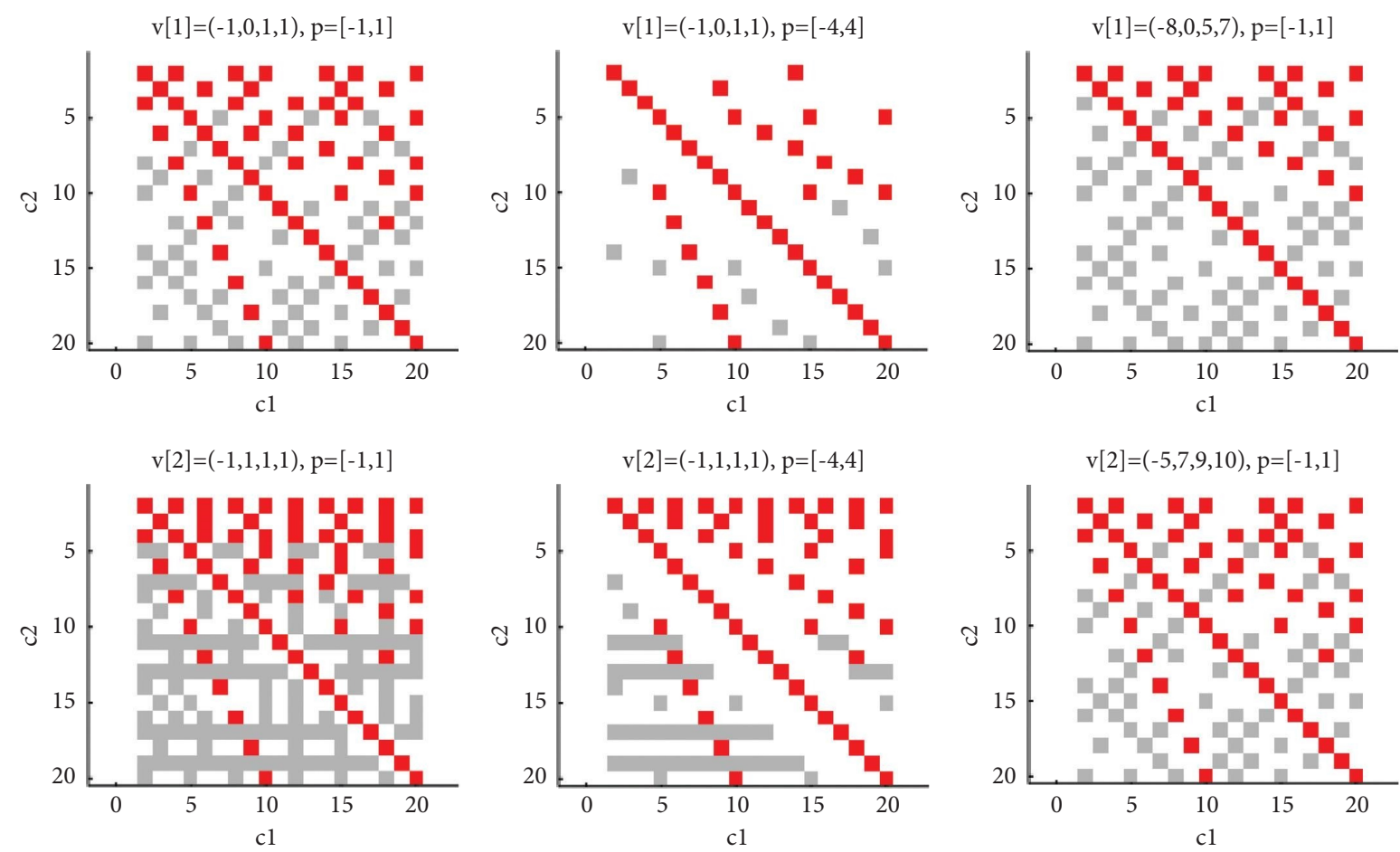

Figure 5: Attractors for tuples $v_{1}$ and $v_{2}$ from Proposition 2. The tuple $v_{1}$ generates symbiosis of the two species; that is, they have to appear at the same time (here, global attractors are again those with $c_{1}=c_{2}$ ). The tuple $v_{2}$ generates a "commensalism" relation: the period $c_{1}$ has to be an integer multiple of $c_{2}, c_{2} \mid c_{1}$, independently of $c_{1}$ (a "bamboo" effect).
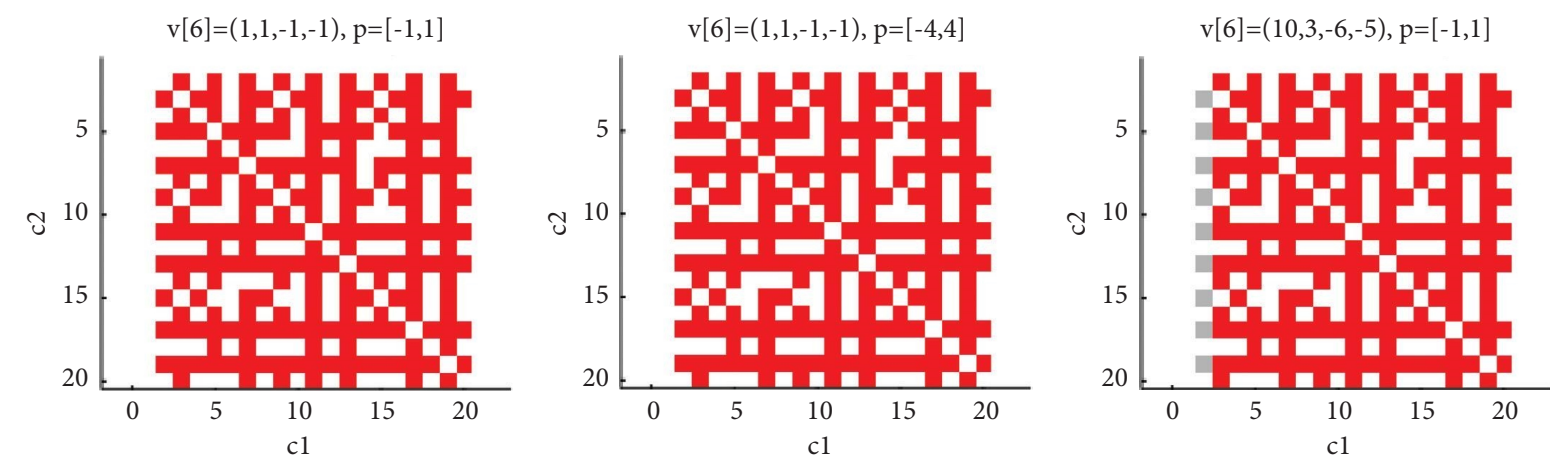

Figure 6: Attractors for tuple $v_{6}$ from Proposition 3. This is the case of two species, which cannot emerge together-they are mutually harmful. In this sense, the competition exclusion principle can originate local extinction in populations. Global fixed points are those with $\operatorname{gcd}\left(c_{1}, c_{2}\right)=1$. Notice that the fixed points for fitness tuples $v_{6}, v_{7}, v_{8}, v_{11}$ and $v_{14}$ are similar.

one. In both cases, there is a "commensalism" interaction, a special kind of symbiosis.

The tuples $v_{1}$ and $v_{2}$ from the class $\mathscr{V}_{2}$ (Proposition 2) generate two types of behavior. The tuple $v_{1}$ generates symbiosis of the two species; that is, they have to appear at the same time. The tuple $v_{2}$ generates a "commensalism" relation: the period $c_{1}$ has to be an integer multiple of $c_{2}$ (which is independent of $c_{1}$ ), so it is also a kind of a "bamboo" effect.

The tuple $v_{6}$ (Proposition 3) is interesting since it is the case of two species that cannot emerge together-they are mutually harmful. In this sense, it is widely known that the competition exclusion principle can originate local extinction in populations [27] and could be represented by this proposition. According to the host-predator hypothesis, the parasitoids, predators, and certain microorganisms specializing on immature stages of a species lack prey or a substrate in the year when only adults are present. The following year, the predators are at low levels when the immature host numbers are again high. The same would hold for specialist predators of adult stage. Several mathematical models have been explored in an attempt to understand the origin of periodicity. Hoppensteadt and Keller [28] showed that synchronized emergence of a single year class is a possible consequence of predation given a limited environmental carrying capacity and life cycle lengths above a certain threshold value. Bulmer [29] also demonstrated that predation alone will not cause periodical behavior 

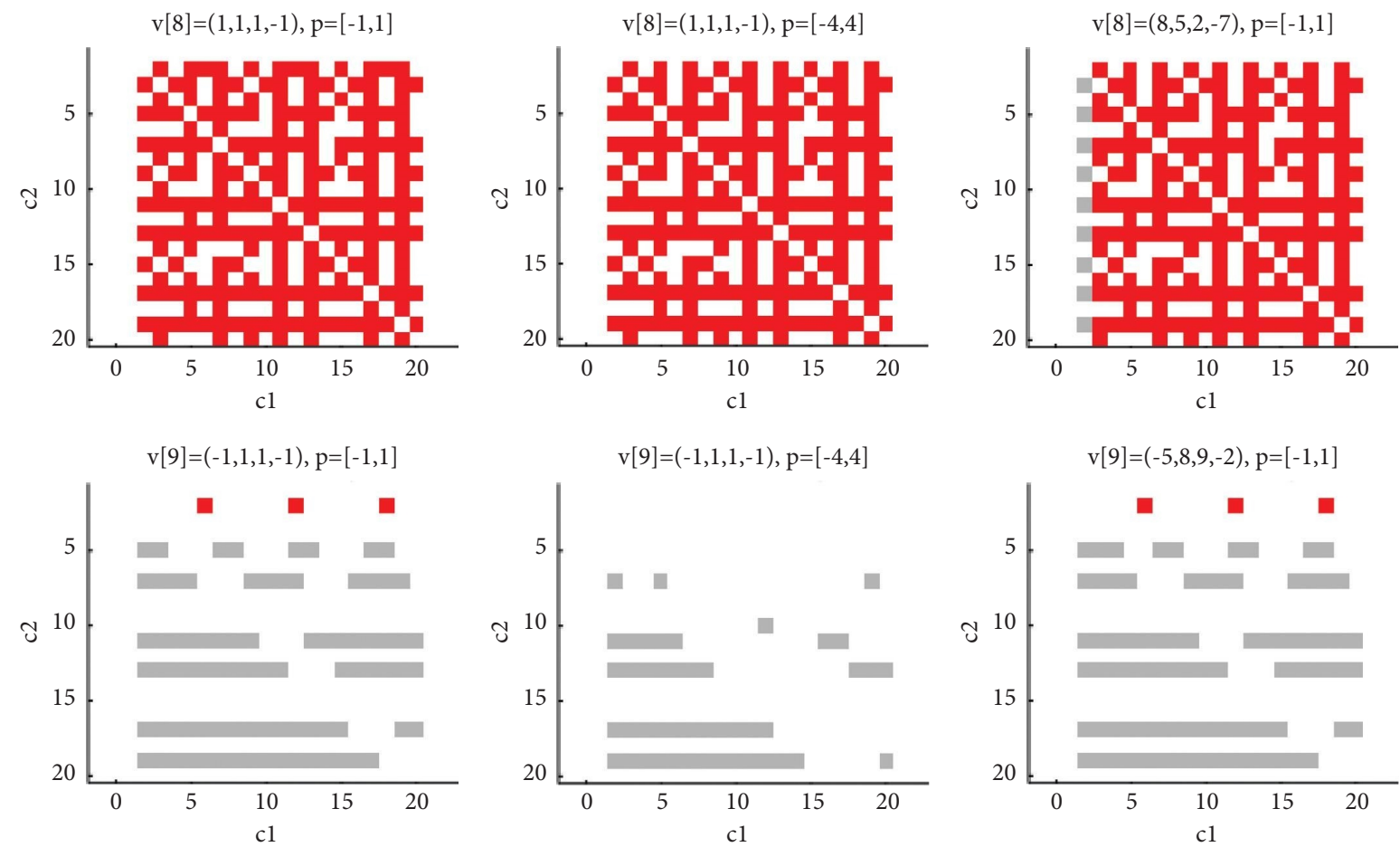

Figure 7: Attractors for tuples $v_{8}$ and $v_{9}$ from Proposition 4. Both tuples generate predator-prey interaction, with the species $\mathscr{C}_{2}$ being the prey. For $v_{8}, \mathscr{C}_{1}$ does not depend on $\mathscr{C}_{2}$ (it may feed on other items), but $\mathscr{C}_{2}$ has to escape; hence, global fixed points are those with $\operatorname{gcd}\left(c_{1}, c_{2}\right)=1$. For $v_{9}$ there is dependence between the prey and the predator: the species $\mathscr{C}_{1}$ must eat $\mathscr{C}_{2}$ in order to survive, and $\mathscr{C}_{2}$ must escape from $\mathscr{C}_{1}$ in order to survive. Local fixed points are those with prime $c_{2}$ and $c_{2} \notin\left\{c_{1}-1, c_{1}+1\right\}$ (cicadas-like behavior). Here, also $F_{1}\left(c_{1}, c_{2}\right)<0$, so the species $\mathscr{C}_{1}$ disappears.
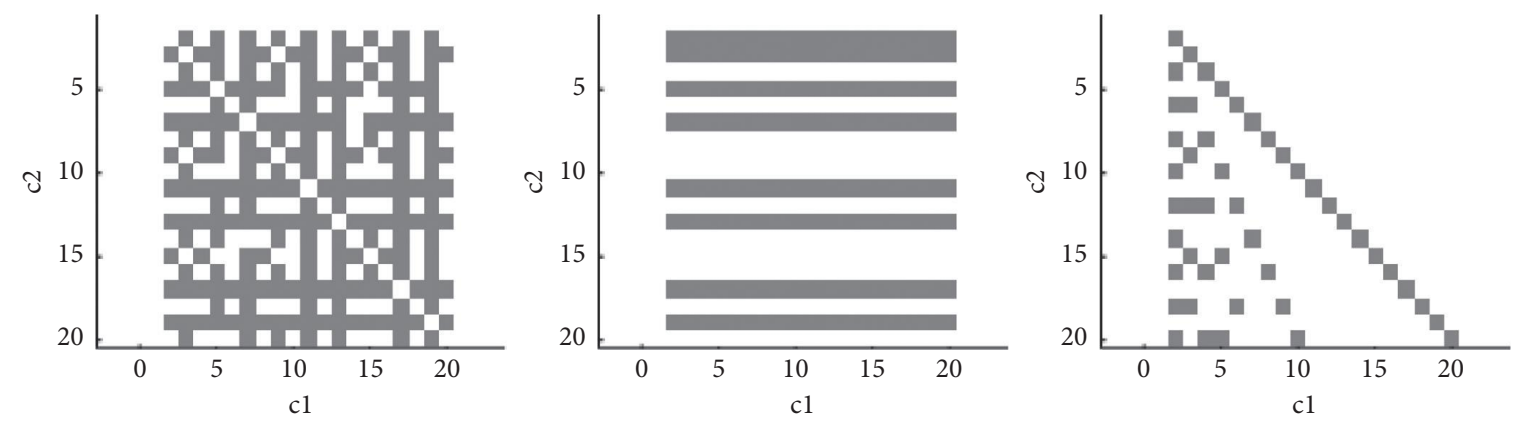

FIGURE 8: For easier visual reference, here are canonical plots of three typical situations: gray denotes pairs $\left(c_{1}, c_{2}\right), c_{1}, c_{2}>1$, such that $\operatorname{gcd}\left(c_{1}, c_{2}\right)=1, c_{2}$ is prime, or $c_{2} \mid c_{1}$, respectively.

except as the accidental result of particular initial conditions. In this sense, our model is concordant with the last reported case.

The tuples $v_{8}$ and $v_{9}$ from the class $\mathscr{V}_{4}$ (Proposition 4) both generate predator-prey interaction, with the species $\mathscr{C}_{2}$ being the prey. For $v_{8}, \mathscr{C}_{1}$ does not depend on $\mathscr{C}_{2}$ (it may feed on other items), but $\mathscr{C}_{2}$ has to escape, hence $\operatorname{gcd}\left(c_{1}, c_{2}\right)=1$. For $v_{9}$ there appears real dependence between the prey and the predator: the species $\mathscr{C}_{1}$ must eat $\mathscr{C}_{2}$ in order to survive, and $\mathscr{C}_{2}$ must escape from $\mathscr{C}_{1}$ in order to survive. In this case, classes of fixed points are those with prime $c_{2}$ in which case $F_{1}\left(c_{1}, c_{2}\right)<0$ so the species $\mathscr{C}_{1}$ disappears. This was the case studied widely in $[20,30]$.

Some open questions and generalizations are related to considering full fitness tuples

$$
v=\left(f_{1}(1,0), f_{2}(0,1), f_{1}(1,1), f_{2}(1,1), f_{1}(0,1), f_{2}(1,0), f_{1}(0,0), f_{2}(0,0)\right),
$$




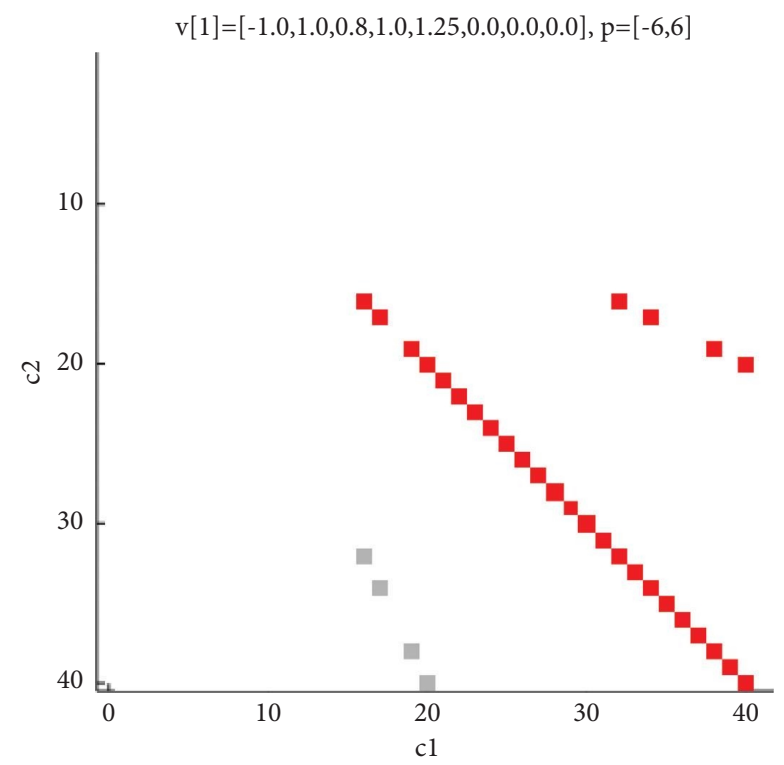

FIGURE 9: Simulation using full fitness tuple with values outside of the set $\{-1,0,1\}$ generates periods longer than 12 for both species, and the species $\mathscr{C}_{1}$ has periods $32,34,38,39$, and 40 , as observed in some species of long-period masting bamboos.

example, large vegetative intervals of bamboos could be explained by the need to minimize energy [31], so, in this case, the "vegetative" values $f_{1}(0,1)$ or $f_{2}(1,0)$ might be positive. In particular, our simulation for the fitness tuple $v=(-1,1,0.8,1,1.25,0,0,0)$ generates fixed points with periods $26,32,34,38,39$, and 40 for the species $\mathscr{C}_{1}$ (see Figure 9), which were observed in some species of longperiod masting bamboos [2, 19]. Also, bamboos periodical life cycles can be dramatically affected during the flowering event by anthropogenic habitat modification that cause delayed reproduction [32]. That is, the combination of a fixed juvenile development time and a long adult life could favor the development of periodical behavior but does not guarantee it [33]. Again, this "qualitative" simulation is by no means proof or explanation of longperiod masting; it is an example of the universality of our theoretical model.

\section{Conclusion}

The presented theoretical model covers all types of periodic behavior. We learned that, from a simple arithmetic point of view, the different interactions are related to only four principles. Given a couple of integer periods $\left(c_{1}, c_{2}\right)$ the principles are as follows:

(i) $\operatorname{gcd}\left(c_{1}, c_{2}\right)=1$ (predation or interspecific competition interaction as for tuples $v_{6}, v_{7}, v_{8}, v_{11}$ and $v_{14}$ )

(ii) One of $c_{1}$ or $c_{2}$ has to be a prime (cicadas-like behavior as for $v_{9}, v_{10}$ and $v_{17}$ )

(iii) $c_{1}=c_{2}$ (symbiosis interaction as for $v_{1}$ and $v_{15}$ ) (iv) One of $c_{1}$ or $c_{2}$ has to be an integer multiple of the other (bamboos-like interaction as for $v_{2}, v_{16}$ and $\left.v_{18}\right)$

Some of these principles are indeed observed in nature-periodical cicadas and long-period masting bamboos.

To be periodical, a species must have a fixed life cycle length, and adults must appear synchronously, reproduce only once, and die [24]. We demonstrate that the emergence of synchrony in some species can occur by chance events, which disrupt this bet-hedging strategy and set the stage for periodicity. Our mathematical model predicts that, given certain initial conditions, intraspecific competition and predation favor its development. These synchronous species should be favored by natural selection later to establish the life cycles according to the interspecific interactions or environment restrictions. Finally, it is established that the variable intensity of interspecific competition, predation, and/or parasitism are interactions, which allow the existence of local stable positive periodical solutions for species such as insects and plants. However, as in any biological system, it is unlikely that one factor operates free from the influence of others.

\section{Appendix}

\section{Proofs}

For the proofs, we need some notations and definitions. We define the following quantities regarding the number of emergences in the interval $[1, T]$ : 
$\left(\mathscr{C}_{1}\right)=$ the number of times species $\mathscr{C}_{1}$ emerges,

$\left(\mathscr{C}_{2}\right)=$ the number of times species $\mathscr{C}_{2}$ emerges,

$\left(\mathscr{C}_{1} \wedge \mathscr{C}_{2}\right)=$ the number of times $\mathscr{C}_{1}$ and $\mathscr{C}_{2}$ emerge in the same year,

$\left(\mathscr{C}_{1} \wedge \overline{\mathscr{C}}_{2}\right)=$ the number of times $\mathscr{C}_{1}$ emerges and $\mathscr{C}_{2}$ does not emerge,

$\left(\overline{\mathscr{C}_{1}} \wedge \mathscr{C}_{2}\right)=$ the number of times $\mathscr{C}_{1}$ does not emerge and $\mathscr{C}_{2}$ emerges.

From $T=c_{1} \cdot c_{2}$, some elementary equalities follow:

$$
\begin{aligned}
\left(\mathscr{C}_{1}\right) & =c_{2}, \\
\left(\mathscr{C}_{2}\right) & =c_{1}, \\
\left(\mathscr{C}_{1} \wedge \mathscr{C}_{2}\right)+\left(\mathscr{C}_{1} \wedge \overline{\mathscr{C}}_{2}\right) & =c_{2}, \\
\left(\mathscr{C}_{1} \wedge \mathscr{C}_{2}\right)+\left(\overline{\mathscr{C}_{1}} \wedge \mathscr{C}_{2}\right) & =c_{1} .
\end{aligned}
$$

Proof. of Lemma 1

For the first six tuples, we have

$$
F_{1}\left(c_{1}, c_{2}\right)=\frac{1}{c_{2}}\left(-\left(\mathscr{C}_{1} \wedge \mathscr{C}_{2}\right)\right) \leq-\frac{1}{c_{2}}<0 .
$$

For the last tuple, we have

$$
F_{2}\left(c_{1}, c_{2}\right)=\frac{1}{c_{1}}\left(-\left(\mathscr{C}_{1} \wedge \mathscr{C}_{2}\right)\right) \leq-\frac{1}{c_{1}}<0 .
$$

\section{Proof. of Lemma 2}

We will only give the proof for fixed points in the class $\mathscr{V}_{4}$; the other proofs are similar. For the tuple $v_{8}=(1,1,-1,1)$, we have

$$
\begin{aligned}
& F_{1}\left(c_{1}, c_{2}\right)=1, \\
& F_{2}\left(c_{1}, c_{2}\right)=\frac{1}{c_{1}}\left(\left(\overline{\mathscr{C}_{1}} \wedge \mathscr{C}_{2}\right)-\left(\mathscr{C}_{1} \wedge \mathscr{C}_{2}\right)\right) .
\end{aligned}
$$

For the tuple $v_{9}=(-1,1,1,-1)$, we have

$$
\begin{aligned}
& F_{1}\left(c_{1}, c_{2}\right)=\frac{1}{c_{2}}\left(-\left(\mathscr{C}_{1} \wedge \overline{\mathscr{C}_{2}}\right)+\left(\mathscr{C}_{1} \wedge \mathscr{C}_{2}\right)\right), \\
& F_{2}\left(c_{1}, c_{2}\right)=\frac{1}{c_{1}}\left(\left(\overline{\mathscr{C}_{1}} \wedge \mathscr{C}_{2}\right)-\left(\mathscr{C}_{1} \wedge \mathscr{C}_{2}\right)\right) .
\end{aligned}
$$

For the tuple $v_{12}=(0,1,1,-1)$, we have

$$
\begin{aligned}
& F_{1}\left(c_{1}, c_{2}\right)=\frac{1}{c_{2}}\left(\mathscr{C}_{1} \wedge \mathscr{C}_{2}\right), \\
& F_{2}\left(c_{1}, c_{2}\right)=\frac{1}{c_{1}}\left(\left(\overline{\mathscr{C}_{1}} \wedge \mathscr{C}_{2}\right)-\left(\mathscr{C}_{1} \wedge \mathscr{C}_{2}\right)\right) .
\end{aligned}
$$

Therefore,

$$
\begin{aligned}
\left(c_{1}, c_{2}\right) \in \mathscr{A}_{8} \Leftrightarrow F_{2}\left(c_{1}, c_{2}^{\prime}\right) & \geq F_{2}\left(c_{1}, c_{2}\right), \\
\left(c_{1}, c_{2}\right) \in \mathscr{A}_{9} \Leftrightarrow F_{1}\left(c_{1}^{\prime}, c_{2}\right) & \geq F_{1}\left(c_{1}, c_{2}\right) \wedge F_{2}\left(c_{1}, c_{2}^{\prime}\right) \geq F_{2}\left(c_{1}, c_{2}\right), \\
\left(c_{1}, c_{2}\right) \in \mathscr{A}_{12} \Leftrightarrow F_{1}\left(c_{1}^{\prime}, c_{2}\right) & \geq F_{1}\left(c_{1}, c_{2}\right) \wedge F_{2}\left(c_{1}, c_{2}^{\prime}\right) \geq F_{2}\left(c_{1}, c_{2}\right) .
\end{aligned}
$$

From that, it is direct $\mathscr{A}_{9}=\mathscr{A}_{12} \subsetneq \mathscr{A}_{8}$. The strictness of the inclusion follows since, for instance, $\left(c_{1}, c_{2}\right)=(3,14) \in \mathscr{A}_{8}$, but $\quad\left(c_{1}, c_{2}\right)=(3,14) \notin \mathscr{A}_{9} \quad$ because $F_{1}(3+1,14)<$ $F_{1}(3,14)$.

Proof. of Proposition 1

For $v_{7}=(1,1,-1,0)$, we have

$$
\begin{aligned}
& F_{1}\left(c_{1}, c_{2}\right)=\frac{1}{c_{2}}\left(\left(\mathscr{C}_{1} \wedge \overline{\mathscr{C}}_{2}\right)-\left(\mathscr{C}_{1} \wedge \mathscr{C}_{2}\right)\right)=\frac{1}{c_{2}}\left(c_{2}-2\left(\mathscr{C}_{1} \wedge \mathscr{C}_{2}\right)\right), \\
& F_{2}\left(c_{1}, c_{2}\right)=\frac{1}{c_{1}}\left(\overline{\mathscr{C}_{1}} \wedge \mathscr{C}_{2}\right)=\frac{1}{c_{1}}\left(c_{1}-2\left(\mathscr{C}_{1} \wedge \mathscr{C}_{2}\right)\right) .
\end{aligned}
$$

Clearly, if $\operatorname{gcd}\left(c_{1}, c_{2}\right)=1$, then

$$
\begin{aligned}
& F_{1}\left(c_{1}, c_{2}\right)=\frac{c_{2}-2}{c_{2}}, \\
& F_{2}\left(c_{1}, c_{2}\right)=\frac{c_{1}-1}{c_{1}},
\end{aligned}
$$

and nobody may improve.

Consider the family

$$
\left(c_{1}, c_{2}\right)=\left(20,16^{n} \cdot 4 \cdot 29 \cdot 31\right)=\left(30,16^{n} \cdot 3596\right) \text {. }
$$

Clearly, $\operatorname{gcd}\left(c_{1}, c_{2}\right)=2, \quad \operatorname{gcd}\left(c_{1}-1, c_{2}\right)=29, \quad$ and $\operatorname{gcd}\left(c_{1}+1, c_{2}\right)=31$ so $F_{1}\left(c_{1} \pm 1, c_{2}\right)<F_{1}\left(c_{1}, c_{2}\right)$, so the species $\mathscr{C}_{1}$ cannot improve its fitness. Let us prove that the species $\mathscr{C}_{2}$ cannot change its period, as well. For that, we will prove that $\forall n \geq 0,5 \mid 16^{n} \cdot 3596-1$ and $3 \mid 16^{n} \cdot 3596+1$ : for $n=0$ clearly $3596-1=3593$ is divisible by 5 , and $3596+1=3597=3 \cdot 1199$. The induction step for $c_{2}-1$ is as follows:

$$
16^{n+1} \cdot 3596-1=16^{n} \cdot 3596 \times 16-1=16^{n} \cdot 3596 \times 15+\left(16^{n} \cdot 3596-1\right)
$$


The first term on the right hand side is divisible by 5 , and the second term is divisible by 5 by the induction hypothesis. Similarly, for $c_{2}+1$, we have

$$
16^{n+1} \cdot 3596+1=16^{n} \cdot 3596 \times 16+1=16^{n} \cdot 3596 \times 15+\left(16^{n} \cdot 3596+1\right) .
$$

The first term on the right hand side is divisible by 3 , and the second term is divisible by 3 by the induction hypothesis. Therefore, $\operatorname{gcd}\left(c_{1}, c_{2}+1\right)=5>\operatorname{gcd}\left(c_{1}, c_{2}\right)$ and $\operatorname{gcd}\left(c_{1}, c_{2}-\right.$ $1)=3>\operatorname{gcd}\left(c_{1}, c_{2}\right)$, so $F_{2}\left(c_{1}, c_{2} \pm 1\right)<F_{2}\left(c_{1}, c_{2}\right)$; hence, all members of the family are local fixed points.

Consider now $v_{10}=(-1,1,1,0)$. Consider, for example,

$$
\left(c_{1}, c_{2}\right)=(30,30 k+4), \quad k \geq 0 .
$$

Then, $\operatorname{gcd}\left(c_{1}, c_{2}\right)=2$ so

$$
\begin{aligned}
& F_{1}\left(c_{1}, c_{2}\right)=\frac{1}{c_{2}}\left(-\left(\mathscr{C}_{1} \wedge \overline{\mathscr{C}_{2}}\right)+\left(\mathscr{C}_{1} \wedge \mathscr{C}_{2}\right)\right)=\frac{-c_{2}+4}{c_{2}} \\
& F_{2}\left(c_{1}, c_{2}\right)=\frac{1}{c_{1}}\left(\overline{\mathscr{C}_{1}} \wedge \mathscr{C}_{2}\right)=\frac{c_{1}-2}{c_{1}}
\end{aligned}
$$

Since $c_{1}+1=31$ and $c_{1}-1=29, \operatorname{gcd}\left(c_{1} \pm 1, c_{2}\right)=1$, so the fitness of $\mathscr{C}_{1}$ cannot be improved. On the other hand, $c_{2}+1=30 k+5$, so $\operatorname{gcd}\left(c_{1}, c_{2}+1\right) \geq 5$ and $F_{2}\left(c_{1}, c_{2}+1\right)$ $<F_{2}\left(c_{1}, c_{2}\right)$. Similarly, $c_{2}-1=30 k+3$, so $\operatorname{gcd}\left(c_{1}, c_{2}+1\right) \geq$ 3 and $F_{2}\left(c_{1}, c_{2}-1\right)<F_{2}\left(c_{1}, c_{2}\right)$. Therefore, fitness of $\mathscr{C}_{2}$ cannot be improved, as well, so $\left(c_{1}, c_{2}\right)=(30,30 k+4)$ is a fixed point for all $k \geq 0$. Furthermore, notice that if $c_{2}>4$, it is always $F_{1}\left(c_{1}, c_{2}\right)<0$, so the species $\mathscr{C}_{1}$ disappears. Also, if $\left(c_{1}, c_{2}\right)$ is such that $c_{1} \notin\left\{c_{2}-1, c_{2}+1\right\}$ and $c_{2}$ is a prime number, then $\left(c_{1}, c_{2}\right)$ is a fixed point and $\mathscr{C}_{1}$ also disappears.

Consider now $v_{11}=(1,0,-1,0)$. We have

$$
\begin{aligned}
& F_{1}\left(c_{1}, c_{2}\right)=\frac{1}{c_{2}}\left(\left(\mathscr{C}_{1} \wedge \overline{\mathscr{C}_{2}}\right)-\left(\mathscr{C}_{1} \wedge \mathscr{C}_{2}\right)\right), \\
& F_{2}\left(c_{1}, c_{2}\right)=0 .
\end{aligned}
$$

If $\operatorname{gcd}\left(c_{1}, c_{2}\right)=1$, then $F_{1}=\left(\left(c_{2}-2\right) / c_{2}\right)$ is the maximum, and they are global fixed points. Further, couples $\left(c_{1}, c_{2}\right)=(30,30 k+4), k \geq 0$, are also local fixed points: because $\operatorname{gcd}\left(c_{1}, c_{2}\right)=2$, we have

$$
\begin{aligned}
& F_{1}\left(c_{1}, c_{2}\right)=\frac{1}{c_{2}}\left(2-\left(c_{2}-2\right)\right)=\frac{-c_{2}+4}{c_{2}}, \\
& F_{2}\left(c_{1}, c_{2}\right)=0 .
\end{aligned}
$$

For $v_{14}=(1,1,1,0)$ we have

$$
\begin{aligned}
& F_{1}\left(c_{1}, c_{2}\right)=1, \\
& F_{2}\left(c_{1}, c_{2}\right)=\frac{1}{c_{1}}\left(\overline{\mathscr{C}_{1}} \wedge \mathscr{C}_{2}\right) .
\end{aligned}
$$

Clearly, all couples $\left(c_{1}, c_{2}\right)$ such that $\operatorname{gcd}\left(c_{1}, c_{2}\right)=1$ are global fixed points, and, like in the previous case for $v_{10}$, the members of the family $\left(c_{1}, c_{2}\right)=(30,30 k+4), k \geq 0$, are local fixed points.

For $v_{17}=(1,-1,-1,0)$, we have

$$
\begin{aligned}
& F_{1}\left(c_{1}, c_{2}\right)=\frac{1}{c_{2}}\left(\left(\mathscr{C}_{1} \wedge \overline{\mathscr{C}_{2}}\right)-\left(\mathscr{C}_{1} \wedge \mathscr{C}_{2}\right)\right), \\
& F_{2}\left(c_{1}, c_{2}\right)=\frac{1}{c_{1}}\left(-\left(\overline{\mathscr{C}_{1}} \wedge \mathscr{C}_{2}\right)\right) .
\end{aligned}
$$

Clearly, global fixed points do not exist: if $F_{1}\left(c_{1}, c_{2}\right)$ is at its maximum, that is, $\left(\mathscr{C}_{1} \wedge \overline{\mathscr{C}_{2}}\right)$ (for instance, if $\left.\operatorname{gcd}\left(c_{1}, c_{2}\right)=1\right)$, then $\mathscr{C}_{2}$ may reply by taking $c_{2}^{\prime}=c_{1}$. Also, if $F_{2}\left(c_{1}, c_{2}\right)$ is at its maximum, that is, $\left(\overline{\mathscr{C}_{1}} \wedge \mathscr{C}_{2}\right)=0$ (so $\left.\left(\mathscr{C}_{1} \wedge \mathscr{C}_{2}\right)=c_{1}\right)$, then $\mathscr{C}_{1}$ may reply by taking $c_{1}^{\prime}=c_{2} \pm 1$ : in that case, $\left(c_{1}^{\prime}, c_{2}\right)=1$ so $F_{1}\left(c_{1}^{\prime}, c_{2}\right)$ is better.

An infinite family of local fixed points is given by couples $\left(c_{1}, c_{2}\right)$ such that $c_{2}<c_{1}-1$ and $c_{1}=p$ is prime: then,

$$
F_{1}\left(p, c_{2}\right)=\frac{1}{c_{2}}\left(\left(c_{2}-1\right)-1\right)=\frac{c_{2}-2}{c_{2}},
$$

which is at its maximum, and $F_{2}\left(p, c_{2} \pm 1\right)=F_{2}\left(p, c_{2}\right)$ because $p$ is prime so it is not divisible by any $c_{2}<p$.

The proofs for $v_{15}, v_{16}$ and $v_{18}$ are analogous.

Proof. of Proposition 2

For $v_{1}=(-1,0,1,1)$ we have

$$
\begin{aligned}
& F_{1}\left(c_{1}, c_{2}\right)=\frac{1}{c_{2}}\left(-\left(\mathscr{C}_{1} \wedge \overline{\mathscr{C}_{2}}\right)+\left(\mathscr{C}_{1} \wedge \mathscr{C}_{2}\right)\right), \\
& F_{2}\left(c_{1}, c_{2}\right)=\frac{1}{c_{1}}\left(\mathscr{C}_{1} \wedge \mathscr{C}_{2}\right) .
\end{aligned}
$$

If $\quad c_{1}=c_{2}, \quad$ then $\quad\left(\mathscr{C}_{1} \wedge \overline{\mathscr{C}_{2}}\right)=0, \quad$ so $F_{1}\left(c_{1}, c_{2}\right)=F_{2}\left(c_{1}, c_{2}\right)=1$, which is a maximum, so they are global fixed points.

Consider now the family $\left(c_{1}, c_{2}\right)$ such that $c_{1}$ and $c_{2}$ are prime numbers and $c_{1} \notin\left\{c_{2}-1, c_{2}+1\right\}$ and $c_{2} \notin$ $\left\{c_{1}-1, c_{1}+1\right\}$ (i.e., they are far enough). Then,

$$
\begin{aligned}
& F_{1}\left(c_{1}, c_{2}\right)=\frac{1}{c_{2}}\left(-\left(c_{2}-1\right)+1\right)=\frac{-c_{2}+2}{c_{2}}, \\
& F_{2}\left(c_{1}, c_{2}\right)=\frac{1}{c_{1}} .
\end{aligned}
$$

Since $c_{1}$ and $c_{2}$ are primes, $c_{1} \pm 1$ and $c_{2} \pm 1$ are not divisors of $c_{2}$ or $c_{1}$, respectively, so fitness functions cannot improve. 
But we have other families, too, which are not necessary primes, for instance, $\left(c_{1}, c_{2}\right)=\left(25, c_{2}\right)$, where

$c_{2} \in\{7+10 r \mid(r \geq 0) \wedge(3 \nmid 7+10 r)\}=\{7+10 r \mid r \geq 0\} \backslash\{27+30 s \mid s \geq 0\}$.

The second set is is the set of numbers divisible by 3 and is contained in the first set since $27+30 s=7+10(3 s+2)$. The difference set is infinite and, by Dirichlet's Theorem, since $\operatorname{gcd}(7,10)=1$, it contains infinitely many prime numbers.

For $v_{2}=(-1,1,1,1)$, we have

$$
\begin{aligned}
& F_{1}\left(c_{1}, c_{2}\right)=\frac{1}{c_{2}}\left(-\left(\mathscr{C}_{1} \wedge \overline{\mathscr{C}_{2}}\right)+\left(\mathscr{C}_{1} \wedge \mathscr{C}_{2}\right)\right), \\
& F_{2}\left(c_{1}, c_{2}\right)=\frac{1}{c_{1}} c_{1}=1 .
\end{aligned}
$$

If $c_{2} \mid c_{1}$, then $\left(\mathscr{C}_{1} \wedge \overline{\mathscr{C}_{2}}\right)=0$ so $F_{1}\left(c_{1}, c_{2}\right)=1$, which is the maximum, so it is a global fixed point.

For $\left(c_{1}, c_{2}\right)=\left(2^{p}, 2^{q}\right), \quad p<q, \quad$ it holds $\operatorname{gcd}$ $\left(c_{1} \pm 1, c_{2}\right)=\operatorname{gcd}\left(2^{p} \pm 1,2^{q}\right)=1$, so

$$
F_{1}\left(c_{1}, c_{2}\right)=\frac{1}{2^{q}}\left(-\left(2^{q}-2^{p}\right)+2^{p}\right)=\frac{1}{2^{q}}\left(2^{p+1}-2^{q}\right) .
$$

So, it does not improve.

\section{Proof. of Proposition 3}

For $v_{6}=(1,1,-1,-1)$, we have

$$
\begin{aligned}
& F_{1}\left(c_{1}, c_{2}\right)=\frac{1}{c_{2}}\left(\left(\mathscr{C}_{1} \wedge \overline{\mathscr{C}_{2}}\right)-\left(\mathscr{C}_{1} \wedge \mathscr{C}_{2}\right)\right), \\
& F_{2}\left(c_{1}, c_{2}\right)=\frac{1}{c_{1}}\left(\left(\overline{\mathscr{C}_{1}} \wedge \mathscr{C}_{2}\right)-\left(\mathscr{C}_{1} \wedge \mathscr{C}_{2}\right)\right) .
\end{aligned}
$$

If $\operatorname{gcd}\left(c_{1}, c_{2}\right)=1$, then

$$
\begin{gathered}
F_{1}\left(c_{1}, c_{2}\right)=\frac{c_{2}-2}{c_{2}}, \\
F_{2}\left(c_{1}, c_{2}\right)=\frac{c_{1}-2}{c_{1}},
\end{gathered}
$$

which is a global fixed point.

The members of the family $\left(c_{1}, c_{2}\right)=\left(30,16^{n} \cdot 3596\right)$ are also local fixed points-the proof is as that for $v_{7}$ in Appendix.

Proof. of Proposition 4

For the tuple $v_{8}=(1,1,1,-1)$ we have

$$
\begin{aligned}
& F_{1}\left(c_{1}, c_{2}\right)=1, \\
& F_{2}\left(c_{1}, c_{2}\right)=\frac{1}{c_{1}}\left(\left(\overline{\mathscr{C}_{1}} \wedge \mathscr{C}_{2}\right)-\left(\mathscr{C}_{1} \wedge \mathscr{C}_{2}\right)\right) .
\end{aligned}
$$

Then, $\left(c_{1}, c_{2}\right)$ such that $\operatorname{gcd}\left(c_{1}, c_{2}\right)=1$ is a global fixed point, since in this case $\left(\mathscr{C}_{1} \wedge \overline{\mathscr{C}}_{2}\right)=\left(\overline{\mathscr{C}}_{1} \wedge \mathscr{C}_{2}\right)=1$, so

$$
F_{2}\left(c_{1}, c_{2}\right)=\frac{1}{c_{1}}\left(\left(c_{1}-1\right)-1\right)=\frac{c_{1}-2}{c_{1}},
$$

is the maximum.

There exist infinitely many local fixed points; for instance,

$$
\left(c_{1}, c_{2}\right)=\left(2 \cdot 3^{b} \cdot 5^{c}, 4\right), \quad b, c \geq 1 .
$$

In this case, $\operatorname{gcd}\left(c_{1}, c_{2}\right)=2$, so

$$
F_{2}\left(c_{1}, c_{2}\right)=\frac{1}{c_{1}}\left(\left(c_{1}-2\right)-2\right)=\frac{c_{1}-4}{c_{1}} .
$$

But $c_{2}-1=3, \quad c_{2}+1=5$, and in both cases $\operatorname{gcd}\left(c_{1}, c_{2} \pm 1\right) \geq 3$, so $F_{2}\left(c_{1}, c_{2} \pm 1\right)<F_{2}\left(c_{1}, c_{2}\right)$.

Consider now $v_{9}=(-1,1,1,-1)$. We have

$$
\begin{aligned}
& F_{1}\left(c_{1}, c_{2}\right)=\frac{1}{c_{2}}\left(-\left(\mathscr{C}_{1} \wedge \overline{\mathscr{C}}_{2}\right)+\left(\mathscr{C}_{1} \wedge \mathscr{C}_{2}\right)\right), \\
& F_{2}\left(c_{1}, c_{2}\right)=\frac{1}{c_{1}}\left(\left(\overline{\mathscr{C}_{1}} \wedge \mathscr{C}_{2}\right)-\left(\mathscr{C}_{1} \wedge \mathscr{C}_{2}\right)\right) .
\end{aligned}
$$

If $\operatorname{gcd}\left(c_{1}, c_{2}\right)=1$, then $\left(\mathscr{C}_{1} \wedge \mathscr{C}_{2}\right)=1$ and

$$
\begin{aligned}
& F_{1}\left(c_{1}, c_{2}\right)=\frac{-c_{2}+2}{c_{2}}, \\
& F_{2}\left(c_{1}, c_{2}\right)=\frac{c_{1}-2}{c_{1}} .
\end{aligned}
$$

If $c_{2}$ is prime and $c_{1} \pm 1 \notin\left\{c_{2} \pm 1\right\}$, then $\left(c_{1}, c_{2}\right)$ is a local fixed point. If, additionally, $c_{2} \geq 3$, the species $\mathscr{C}_{1}$ disappears.

But there exist infinitely many local fixed points. Let us see one case: consider $\left(c_{1}, c_{2}\right)=(2 \cdot 3 \cdot 5,2 s)=(30,2 s)$ such that $3 \mid\left(c_{2}-1\right)$ and $5 \mid\left(c_{2}+1\right)$. Clearly, we have $\operatorname{gcd}(30,2 s)=2$, so

$$
\begin{aligned}
& F_{1}\left(c_{1}, c_{2}\right)=\frac{1}{c_{2}}\left(-\left(c_{2}-2\right)+2\right)=\frac{-c_{2}+4}{c_{2}}, \\
& F_{2}\left(c_{1}, c_{2}\right)=\frac{1}{c_{1}}\left(\left(c_{1}-2\right)-2\right)=\frac{c_{1}-4}{c_{1}} .
\end{aligned}
$$

But, since $c_{1}-1=29$ and $c_{1}+1=31$ are both prime numbers,

$$
\begin{aligned}
F_{1}\left(c_{1} \pm 1, c_{2}\right) & =\frac{1}{c_{2}}\left(-\left(c_{2}-1\right)+1\right)=\frac{-c_{2}+2}{c_{2}}<\frac{-c_{2}+4}{c_{2}} \\
& =F_{1}\left(c_{1}, c_{2}\right),
\end{aligned}
$$

so the fitness of the species $\mathscr{C}_{1}$ cannot improve.

Let us see $c_{2}: 2 s \pm 1$ is not even and, by hypothesis, it is divisible by 3 or 5 . Therefore, $\operatorname{gcd}\left(c_{1}, c_{2} \pm 1\right) \geq 3$ so $F_{2}\left(c_{1}, c_{2} \pm 1\right)<F_{2}\left(c_{1}, c_{2}\right)$, and the fitness of the species $\mathscr{C}_{2}$ cannot improve either.

It remains to prove that there are infinitely many prime numbers $s$ such that $3 \mid(2 s-1)$ and $5 \mid(2 s+1)$. Suppose 


$$
\begin{aligned}
c_{2}-1 & =2 s-1=3 m, \\
c_{2}+1 & =2 s+1=5 n, \\
m, n & \in \mathbb{N} .
\end{aligned}
$$

Then, $3 m+2=5 n$, so

$$
m=\frac{5 n-2}{3}=\frac{(3+2) n-2}{3}=n+2\left(\frac{n-1}{3}\right), \quad n \in \mathbb{N} \text {. }
$$

There exist integer solutions for $m$ and $n$ : for $n=4+3 k$, $k \in \mathbb{N}, k \geq 0$ it holds that

$$
m=\frac{5(4+3 k)-2}{3}=\frac{18+15 k}{3}=5 k+6 .
$$

Then,

$$
2 s=3 m+1=3(5 k+6)+1=15 k+19, \quad k \geq 0 .
$$

Since $2 s$ is even, $k$ must be odd. By setting $k=2 l+1$, $l \geq 0$, we have

$$
c_{2}=2 s \in\{34+30 l \mid l \geq 0\} \equiv B .
$$

Set $B$ is an arithmetic progression such that $\operatorname{gcd}(17,15)=1$; hence, by Dirichlet's Theorem, it contains infinitely many primes. We conclude that there exist infinitely many couples $(30,2 s), s$ prime, which are local fixed points for the rule $v_{9}$. Since $c_{2}>4$, we have $F_{1}\left(c_{1}, c_{2}\right)<0$, so the species $\mathscr{C}_{1}$ disappears.

\section{Data Availability}

In this research, no data were used. Instead, simulations were performed using computer program. The complete program used to run simulations and produce figures is available at https:/github.com/ivanslapnicar/ EvolutionaryModel.jl

\section{Disclosure}

A preprint of the article is available on arXiv at https://arxiv. org/abs/2010.00940.

\section{Conflicts of Interest}

The authors declare that they have no conflicts of interest.

\section{Acknowledgments}

Research of E. Goles was funded by the grant FondecytANID 1200006. Research of I. Slapničar was supported in part by Croatian Science Foundation under the project IP2020-02-2240. M. Lardies acknowledges the support of the grants Fondecyt-ANID 1190444 and ANID-Millennium Science Initiative Program-CODE ICN2019_015. Part of this work was done while E. G. was visiting University of Split. Part of this work was done while I. S. was visiting Universidad Adolfo Ibañez supported by the grant from Centro de Modelamiento Matematico, Santiago, Chile. The authors would like to thank James Powell, Utah State
University, Logan, for commenting on the final draft of the paper.

\section{References}

[1] D. A. Roff, Life History Evolution, Sinauer Associates, Sunderland, MA, USA, 2002.

[2] D. H. Janzen, "Why bamboos wait so long to flower," Annual Review of Ecology and Systematics, vol. 7, no. 1, pp. 347-391, 1976.

[3] L. L. Rockwood, Introduction to Population Biology, Wiley \& Blackwell, Oxford, UK, 2015.

[4] M. Lloyd and H. S. Dybas, "The periodical cicada problem. II. Evolution," Evolution, vol. 20, no. 4, pp. 466-505, 1966.

[5] R. May, "Parasitic infections as regulators of animal populations: the dynamic relationship between parasites and their host populations offers clues to the etiology and control of infectious disease," The American Naturalist, vol. 71, pp. 36-45, 1983.

[6] P. Grant, "The priming of periodical cicada life cycles," Trends in Ecology \& Evolution, vol. 20, no. 4, pp. 169-174, 2005.

[7] D. E. Beasley, C. A. Penick, N. S. Boateng, H. L. Menninger, and R. R. Dunn, "Urbanization disrupts latitude-size rule in 17-year cicadas," Ecology and Evolution, vol. 8, no. 5, pp. 2534-2541, 2018.

[8] R. E. Ricklefs, "Disintegration of the ecological community," The American Naturalist, vol. 172, no. 6, pp. 741-750, 2008.

[9] M. Y. Chen, "Giant timber bamboo in Alabama," Journal of Forestry, vol. 71, p. 777, 1973.

[10] W. D. Koenig and A. M. Liebhold, "Avian predation pressure as a potential driver of periodical cicada cycle length," The American Naturalist, vol. 181, no. 1, pp. 145-149, 2013.

[11] C. Goodnight, E. Rauch, H. Sayama, M. A. M. De Aguiar, M. Baranger, and Y. Bar-yam, "Evolution in spatial predatorprey models and the prudent predator: the inadequacy of steady-sate organism fitness and the concept of individual and group selection," Complexity, vol. 13, pp. 23-44, 2008.

[12] S. Kakishima, J. Yoshimura, H. Murata, and J. Murata, "6-year periodicity and variable synchronicity in a mass-flowering plant," PLoS One, vol. 6, no. 12, Article ID e28140, 2011.

[13] J. Travis, J. Leips, and H. F. Rodd, "Evolution in population parameters: density-dependent selection or density-dependent fitness?" The American Naturalist, vol. 181, pp. s9-s20, 2013.

[14] Y. Tanaka, J. Yoshimura, C. Simon, J. R. Cooley, and K.-i. Tainaka, "Allee effect in the selection for prime-numbered cycles in periodical cicadas," Proceedings of the National Academy of Sciences, vol. 106, no. 22, pp. 8975-8979, 2009.

[15] D. Kelly and V. L. Sork, "Mast seeding in perennial plants: why, how, where?" Annual Review of Ecology and Systematics, vol. 33, no. 1, pp. 427-447, 2002.

[16] J. Yoshimura, "The evolutionary origins of periodical cicadas during ice ages," The American Naturalist, vol. 149, no. 1, pp. 112-124, 1997.

[17] J. Yoshimura, T. Hayashi, Y. Tanaka, K.-i. Tainaka, and C. Simon, "Selection for prime-number intervals in a numerical model of periodical cicada evolution," Evolution, vol. 63, no. 1, pp. 288-294, 2009.

[18] H. Ito, S. Kakishima, T. Uehara et al., "Evolution of periodicity in periodical cicadas," Scientific Reports, vol. 5, no. 1, Article ID 14094, 2015.

[19] C. Veller, M. A. Nowak, and C. C. Davis, "Extended flowering intervals of bamboos evolved by discrete multiplication," Ecology Letters, vol. 18, no. 7, pp. 653-659, 2015. 
[20] E. Goles, O. Schulz, and M. Markus, "Prime number selection of cycles in a predator-prey model," Complexity, vol. 6, no. 4, pp. 33-38, 2001.

[21] S. C. Stearns, The Evolution of Life Histories, Oxford University Press, London, UK, 1992.

[22] J. D. O’Sullivan, J. C. D. Terry, and A. G. Rossberg, "Intrinsic ecological dynamics drive biodiversity turnover in model metacommunities," Nature Communications, vol. 12, p. 3627, 2021.

[23] M. C. Urban, S. Y. Strauss, F. Pelletier et al., "Evolutionary origins for ecological patterns in space," Proceedings of the National Academy of Sciences, vol. 117, no. 30, pp. 1748217490, 2020.

[24] K. Heliövaara, R. Väisänen, and C. Simon, "Evolutionary ecology of periodical insects," Trends in Ecology \& Evolution, vol. 9, no. 12, pp. 475-480, 1994.

[25] S. R. Hajong and S. Yaakop, "Chremistica ribhoi sp. n. (Hemiptera: cicadidae) from north-east India and its mass emergence," Zootaxa, vol. 3702, no. 5, pp. 493-500, 2013.

[26] C. Guerreiro, "Flowering cycles of woody bamboos native to southern South America," Journal of Plant Research, vol. 127, no. 2, pp. 307-313, 2014.

[27] J. Bengtsson, "Interspecific competition increases local extinction rate in a metapopulation system," Nature, vol. 340, no. 6236, pp. 713-715, 1989.

[28] F. Hoppensteadt and J. Keller, "Synchronization of periodical cicada emergences," Science, vol. 194, no. 4262, pp. 335-337, 1976.

[29] M. G. Bulmer, "Periodical insects," The American Naturalist, vol. 111, no. 982, pp. 1099-1117, 1977.

[30] E. Goles, O. Schulz, and M. Markus, "A biological generator of prime numbers," Nonlinear Phenomena in Complex Systems, vol. 3, pp. 208-213, 2000.

[31] M. Gadgil and W. H. Bossert, "Life historical consequences of natural selection," The American Naturalist, vol. 104, pp. 1-24, 1970.

[32] W. Wang, S. B. Franklin, Z. Lu, and B. J. Rude, "Delayed flowering in bamboo: evidence from Fargesia qinlingensis in the Qinling mountains of China," Frontiers in Plant Science, vol. 7, no. 151, p. 151, 2016.

[33] M. Lauzon and P. P. Harper, "Life history and production of the stream-dwelling mayfly Habrophlebia vibrans Needham (Ephemeroptera; Leptophlebiidae)," Canadian Journal of Zoology, vol. 64, no. 9, pp. 2038-2045, 1986. 